4 Packe GE, Ayres JG, Citron KM, et al. Large lung bullae in sarcoidosis. Thorax 1986; 41: 792-797.

5 Pena CM, Cosgrove DM, Eng P, et al. Bullectomies for bullous sarcoidosis. Cleve Clin J Med 1993; 60: 157-160.

6 Froudarakis ME, Bouros D, Voloudaki A, et al. Pneumothorax as a first manifestation of sarcoidosis. Chest 1997; 112: 278-280.

7 Judson MA, Strange C. Bullous sarcoidosis: a report of three cases. Chest 1998; 114: 1474-1478.

8 Kumar PD, Epstein DL. Eleven-year survival of a patient with bullous sarcoidosis after bilateral pleurodesis. Am J Med 2001; 111: 165.
9 Judson MA, Vincent B, Huggins T, et al. Unusual presentations of pulmonary sarcoidosis: cases from the medical university of South Carolina. Semin Respir Crit Care Med 2007; 28: 75-82.

10 Mireles-Cabodevilla E, Sahi H, Farver C, et al. A young patient with a minimal smoking history presents with bullous emphysema and recurrent pneumothorax. Chest 2007; 132: 338-343.

11 Keller CA, Naunheim KS, Osterloh J, et al. Histopathologic diagnosis made in lung tissue resected from patients with severe emphysema undergoing lung volume reduction surgery. Chest 1997; 111: 941-947.

\title{
Reproducibility and reliability of pleural fluid cytokine measurements
}

\section{To the Editors:}

Cytokine levels in pleural fluid (PF) have been the subject of many reports focusing on their aetiological, diagnostic and prognostic roles; furthermore, cytokine levels are of importance in translational research of pleural diseases [1]. However, data of these studies were based on a single one-off measurement of cytokines in stored PF samples assayed after thawing. Cytokine concentration is a delicate balance of systemic and lung filtration of cytokines, and release from local (mesothelial and inflammatory and/or cancer) cells [2]. Reproducibility of PF cytokine measurements in humans has not previously been established. Our study aimed to: 1) determine short- and long-term changes of PF cytokine concentrations in patients with recurrent/persistent pleural effusions; and 2) examine the influence of freeze-thaw cycles on cytokine levels.

In total, 137 serial PF samples were obtained from 30 patients who required an indwelling pleural catheter $(n=19)$ or repeated pleural aspirations $(n=11)$ for management of malignant $(n=24)$ or benign $(n=6)$ recurrent effusions. 26 of these patients had PF collected as part of a separate study on the use of PF mesothelin [3]. Additional samples were collected from seven patients (malignant, $n=5$; benign, $n=2$ ) to assess the effects of storage and freeze-thaw cycles. Short-term reproducibility was assessed between paired PF samples taken from patients within any 10-day period. Multiple paired samples were included from patients who had repeated sampling. PF samples were collected and centrifuged as previously published. Supernatants were stored at $-80^{\circ} \mathrm{C}$ until assayed. Vascular endothelial growth factor (VEGF), interleukin (IL)-8, tumour necrosis factor (TNF)- $\alpha$, macrophage chemoattractant protein (MCP)-1, and transforming growth factor (TGF)- $\beta 1$ and TGF- $\beta 2$ concentrations were measured using commercially available ELISA kits (PeproTech EC Ltd, London, UK). Total TGF- $\beta$ levels were measured by activating the PF samples with $\mathrm{HCl}$, followed by neutralisation with hydroxyethyl piperazine ethane sulfonic acid- $\mathrm{NaOH}$ before ELISA assaying, as recommended by the manufacturer. Analyses were repeated on all paired serial samples showing a more than two-fold change for confirmation.

The short-term reproducibility of cytokine measurements in paired samples $(n=46)$ taken within 10 days was excellent (table 1). No significant changes were observed in the cytokines tested (all $\mathrm{p} \geqslant 0.05$ ). Likewise, PF cytokine concentrations remained stable during each patient's disease course. There was no significant change over time in all cytokines

TABLE 1 Cytokine measurements in 46 paired samples taken within 10 days

\begin{tabular}{|c|c|c|c|c|}
\hline & Baseline sample $\mathbf{n g} \cdot \mathrm{mL}^{-1}$ & Paired sample $\mathbf{n g} \cdot \mathrm{mL}^{-1}$ & Variation of paired samples $\mathrm{ng} \cdot \mathrm{mL}^{-1}$ & p-value ${ }^{\#}$ \\
\hline VEGF & $0.53(0.16-2.10)$ & $0.40(0.16-2.16)$ & $0.00 \pm 0.90$ & 0.831 \\
\hline MCP-1/CCL2 & $0.80(0.45-2.01)$ & $0.85(0.55-2.69)$ & $-0.66 \pm 3.12$ & 0.076 \\
\hline TGF- $\beta 1$ & $7.72(5.57-17.14)$ & $6.82(5.15-15.01)$ & $0.90 \pm 8.26$ & 0.965 \\
\hline TGF- $\beta 2$ & $0.08(0.03-0.23)$ & $0.07(0.03-0.20)$ & $0.07 \pm 0.23$ & 0.050 \\
\hline
\end{tabular}



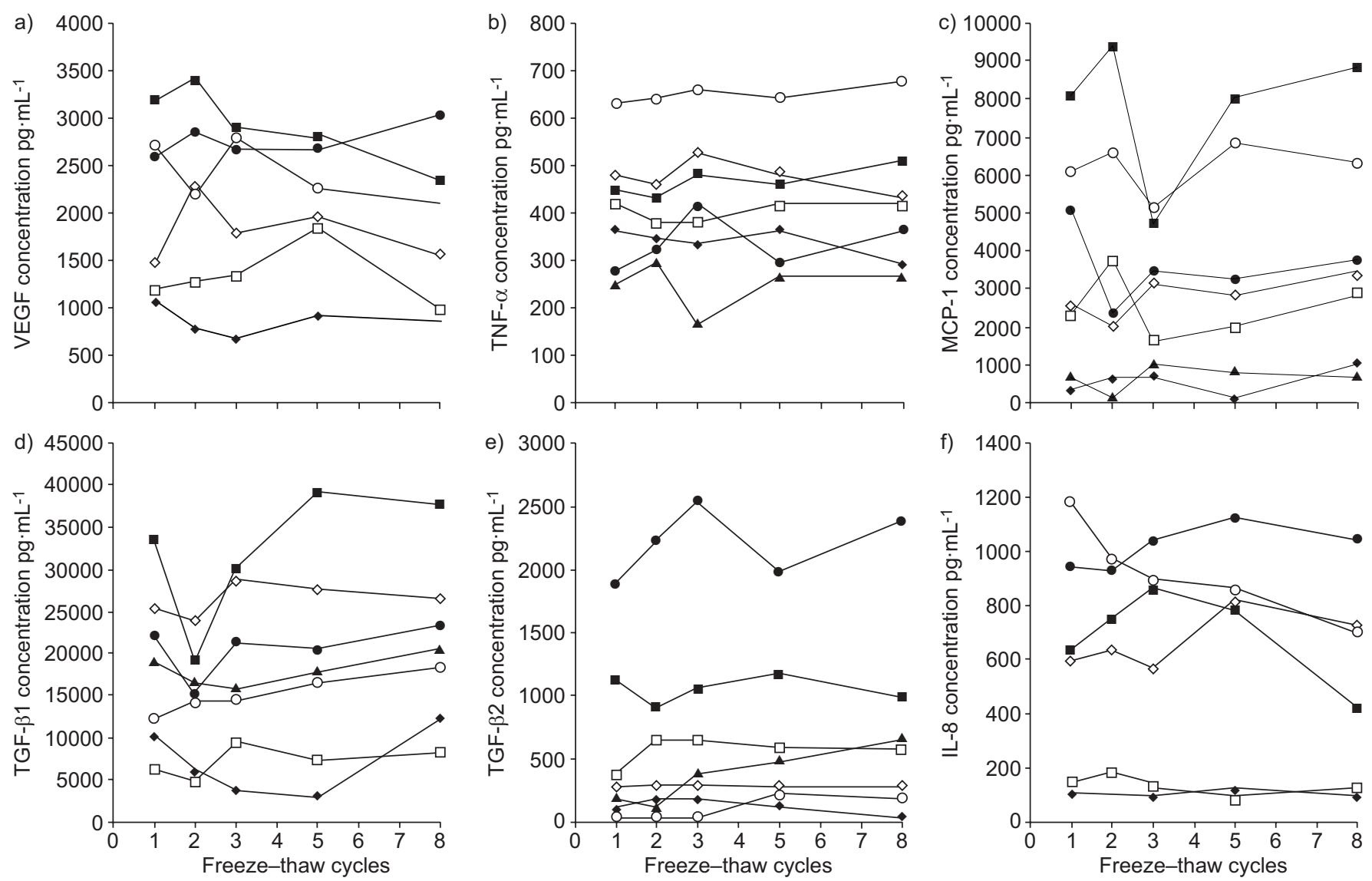

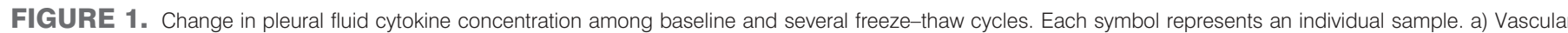

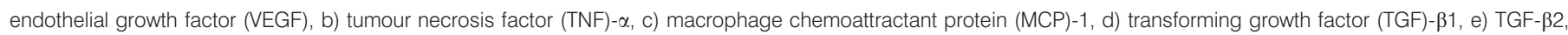
and f) interleukin (IL)-8.

tested from samples obtained at intervals 1-242 days apart (median 45 days). Cytokine levels correlated significantly with each other, in particular, the inter-relationships between VEGF, MCP-1, IL-8 and TGF- $\beta 1$ were highly significant (all $\mathrm{p}<0.001$ ). No obvious differences were noted between malignant and benign samples (data not shown).

To assess the stability of cytokines during the freeze-thaw cycle, PF samples were immediately placed into multiple aliquots after centrifugation and stored at $-80^{\circ} \mathrm{C}$. One aliquot was thawed (to $0^{\circ} \mathrm{C}$ on ice or to room temperature for $2 \mathrm{~h}$ ) and frozen again at $-80^{\circ} \mathrm{C}$ for $\geqslant 6 \mathrm{~h}$. For each $\mathrm{PF}$ sample, separate aliquots were frozen then thawed for a total of one, two, three and eight times and analysed simultaneously. The absolute percentage change in cytokine levels (eight cycles versus baseline) was as follows (mean $\pm \mathrm{SD}$ ). VEGF: $6.76 \pm 6.98 \%$ $(p=0.59) ; \quad$ MCP-1: $25.33 \pm 27.84 \% \quad(p=0.66) ; \quad$ TNF- $\alpha$ : $18.05 \pm 20.45 \%(p=0.63)$; TGF- $\beta 1: 11.6 \pm 13.75 \%(p=0.35)$; TGF$\beta 2: 74.93 \pm 88.48 \%(p=0.66)$; and IL-8: $10.08 \pm 7.23 \%(p=0.54)$. The direction of change varied in all cases with no suggestion of overall reduction or increase in values with repeat freezethaw cycles (fig. 1). Similarly, no significant changes were observed in samples defrosted to $0^{\circ} \mathrm{C}$ only (data not shown).

This study shows, for the first time, that measurements of commonly tested cytokines in PF are highly reproducible, and are stable following the repeated freeze-thaw cycles necessary in the practical handling of PF samples. This provides crucial validation for research studies involving PF cytokine quantification and their potential clinical application. Moreover, the measurements of these cytokines are not dependent on the time during the disease course, which adds strength to the research on diagnostic use of these cytokines.

It is intriguing that the cytokine levels are remarkably stable in the pleural cavity despite the many potential influencing factors, e.g. cell release and metabolism, serum extravasation and systemic reabsorption. It is logical then to assume they are tightly regulated, even in the disease states, for important biological reasons and testify to the current belief that these cytokines hold vital roles in disease biology.

There are limitations to our study. Most of the samples studied were malignant pleural effusions, reflecting that they are the most common cause of recurrent effusions. While it is likely that the reproducibility and stability of the cytokines shown in our samples will extend to recurrent or persistent effusions of other aetiologies, and to other cytokines, this requires confirmation.

\section{S. Bielsa ${ }^{*, \#}$, H.E. Davies ${ }^{\tau,+}$, R.J.O. Davies ${ }^{\tau,+}$, A. Taylor ${ }^{*}$ and Y.C.G. Lee ${ }^{*, \tau,+, \S}$}


*Centre for Respiratory Research, University College London, London, "Oxford Centre for Respiratory Medicine, ${ }^{+}$University of Oxford, Oxford, UK, "Dept of Internal Medicine, Arnau de Vilanova University Hospital, Institut de Reçerca Biomèdica de Lleida, Lleida, Spain, and ${ }^{5}$ Dept of Medicine, University of Western Australia, Perth, Australia.

Correspondence: Y.C.G. Lee, Dept of Medicine and Lung Institute of Western Australia, University of Western Australia, G Block, 4/F, Queen Elizabeth II Medical Centre, Perth WA6009, Australia. E-mail: gary.lee@uwa.edu.au

Support Statement: Y.C.G. Lee received funding from the Medical Research Council (London, UK), the National Health and Medical Research Council (Canberra, Australia) and received the Dept of Health Clinical Senior Lecturer Award (UK Clinical Research Collaboration, London, UK). The Instituto de Salud Carlos III (FIS CM07/00020) provided funding to S. Bielsa. The British Lung Foundation provided funding to H.E. Davies, R.J.O. Davies and Y.C.G. Lee, and funding was given to R.J.O. Davies from the Oxford National Institute of Health Research Biomedical Research Centre Programme (Oxford, UK).

Statement of Interest: None declared.

\section{REFERENCES}

1 Light RW, Lee YCG. Textbook of Pleural Diseases. 2nd Edn. London, Hodder Arnold, 2008.

2 Mutsaers SE, Prele CM, Brody AR, et al. Pathogenesis of pleural fibrosis. Respirology 2004; 9: 428-440.

3 Davies HE, Sadler RS, Bielsa S, et al. The clinical impact and reliability of pleural fluid mesothelin in undiagnosed pleural effusions. Am J Respir Crit Care Med 2009; [Epub ahead of print PMID: 19299498]. 